\title{
Dietmar von der Pfordten, Dignidad humana
}

(2020) Atelier Barcelona, $169 \mathrm{pp}$.

\author{
Jesús Ignacio Delgado Rojas \\ Universidad Carlos III de Madrid \\ ORCID ID 0000-0002-3818-5990 \\ jesusignacio.delgado@uc3m.es
}

Cita recomendada:

Delgado Rojas, J.I. (2020). Dietmar von der Pfordten, Dignidad humana. Eunomía. Revista en Cultura de la Legalidad, 20, pp. 496-501.

doi: https://doi.org/10.20318/eunomia.2021.6093

Aparece en España el libro Dignidad humana del filósofo alemán Dietmar von der Pfordten. La obra va precedida de un esclarecedor estudio preliminar a cargo del profesor José Antonio Santos Arnaiz, a quién debemos el esfuerzo de introducir el pensamiento jurídico-filosófico de Von der Pfortdten en el mundo de habla hispana. La traducción, a partir del trabajo original alemán-Menschenwürde, publicado en 2016-, se debe al doctorando de la Universidad de Göttingen Carlos Alberto Mendoza Cruz. El libro lo edita el sello catalán Atelier en un formato pequeño y compacto.

Dietmar von der Pfordten -1964, Múnich- es, desde 2002, profesor de Filosofía jurídica y social en la Universidad Georg-August de Göttingen. Anteriormente había ocupado la cátedra de Filosofía del derecho y Filosofía social en la Universidad de Erfurt. Se doctoró en Derecho en 1991 bajo la dirección de Arthur Kaufmann. En 1993 se integra en el Centro Internacional de Ética en las Ciencias (IZEW) de la Universidad de Tübingen y en 1994 realiza su tesis doctoral en Filosofía con el profesor Julian Nida-Rümelin -que había sucedido a Günter Patzig al frente de la cátedra-. Von der Pfordten ha sido profesor invitado por Harvard, Columbia y Nueva York; también por las universidades de Groningen, Cagliari y Rey Juan Carlos en España. Es miembro de la Academia de Ciencias de Thüringer de Erfurt y, desde 2003, miembro de la Comisión Asesora del Gobierno Federal de Alemania para la devolución a los judíos de los bienes culturales que les fueron confiscados durante la persecución nazi. 
El libro de Von der Pfordten es un alegato que reivindica la grandeza de la dignidad humana, su historia de éxito, su fuerza moral, su estatus específico en el derecho, su función de fundamento de los derechos humanos y la especial protección de la que goza frente a los ataques que intentan vulnerarla. Asemeja Von der Pfordten la dignidad humana a un corredor que, «habiendo empezado el último, terminó por rebasar a todos sus competidores». Y es que el tardío concepto de la dignidad humana en la historia de la filosofía moral ha tomado finalmente la delantera a todos los derechos humanos, convirtiéndose en «el supremo mandamiento de la moralidad, así como de varias constituciones y acuerdos internacionales» (Von der Pfordten, 2020, p. 21).

Para Von der Pfordten, la dignidad humana tiene como núcleo la autodeterminación sobre los propios intereses y la esencial posición social del individuo. El singular análisis de la dignidad en esta obra se debe a que su tratamiento se encuentra articulado en base a cuatro 'subconceptos' de dignidad humana: uno 'grande', uno 'pequeño', uno 'intermedio' y otro 'económico'.

«Por gran dignidad humana se designa aquel atributo humano incorpóreo, interno, necesario, universal e inmutable en su núcleo, como apareció, aunque de forma todavía imperfecta, ya en Cicerón y, sobre todo, desarrollado durante el Cristianismo y que después, tras varios ensayos durante el Renacimiento en Italia, sería concretado en Kant como autolegislación o autodeterminación. Esta gran dignidad se entiende mejor -así en la propuesta que aquí se somete- como autodeterminación sobre los intereses propios. Por pequeña dignidad se entiende, por el contrario, el atributo incorpóreo, externo y mutable de la relevante posición social y el mérito de una persona tal y como, circunscritos a una determinada posición social, eran ya designados con la expresión latina dignitas. Como caso límite de la pequeña dignidad se conoce desde Pufendorf, además, una dignidad intermedia. También ella se refiere al atributo externo de la relevante posición social de las personas. Acentúa, sin embargo, la natural y, por ello, en principio, inmutable igualdad de la posición social de toda persona. Por último, en el siglo XIX, especialmente los representantes del movimiento socialista, exigieron una "existencia digna". Con ello se demandaba la realización de condiciones económicas o materiales de la dignidad humana. En este sentido, se puede hablar, de forma resumida, de una dignidad económica, más exactamente, de una condición económica de la dignidad» (pp. 25-26).

Ya desde la Introducción Von der Pfordten nos hace partícipes de esta diferenciación que va a manejar. El desarrollo posterior de su trabajo transita por estas cuatro vertientes de la dignidad y muestra cómo los órdenes normativos de la moral, el derecho y la política interaccionan y protegen el estatuto que implica cada modalidad de la dignidad. Y ello manteniendo siempre una concepción de la dignidad entendida como autonomía moral del sujeto, muy cercana a la filosofía kantiana, como atributo humano de carácter interno, incorpóreo, universal e inmutable en su núcleo.

El libro se estructura en cuatro capítulos. El primero de ellos es una introducción que delimita el objeto de la investigación. A continuación, en el capítulo segundo, realiza Von der Pfordten un análisis histórico y jurídico-filosófico de la dignidad humana, a través de sus distintas épocas y pensadores. El tercer capítulo se dedica a exponer las distintas concepciones de la dignidad humana a partir de cuestiones relativas a sus problemas de fundamentación, intangibilidad o universalidad, entre otros. En el cuarto capítulo Von der Pfordten pasa revista a una serie de casos actuales y controvertidos en los que la dignidad humana ocupa un papel protagonista y que son analizados tanto con jurisprudencia alemana y europea como con el enfoque filosófico de los cuatro 'tipos' de dignidad que preside la obra.

No es en la Antigüedad griega, sino en la romana, donde aparece en la historia del pensamiento un concepto de dignidad, externo y mutable, y ligado a la posición 
social, sobre todo política. Se vincula el estatus, la jerarquía en sociedad o el rango que se ocupe en un estamento con la noción de dignidad. Son estas clases sociales, y la categoría que en ellas se detente, las que determinan la dignidad de la persona. En función del lugar ocupado por el sujeto en la pirámide social, se atribuye una dignitas diferente. Al senador y al patricio, al burgués y al siervo, al propietario y al campesino, se les reconoce una dignidad más o menos cualificada. Frente a este criterio propio de la Antigüedad romana -dignitas- y la teología medieval, se abre paso la idea de dignidad cuando el 'valor igual' de cada hombre se genera desde la propia persona, es decir, como propiedad inmanente y presente por igual en cada ser humano. A este recorrido histórico de la idea de dignidad humana dedica Von der Pfordten el primer capítulo. En los siglos posteriores, «este exigente concepto de dignidad humana se consolidó gracias a la filosofía y teología cristianas» (p. 24). Durante la modernidad, el concepto de dignidad alcanzará su auténtico y pujante significado en el Renacimiento Italiano así como en Alemania con Samuel von Pufendorf y, en especial, con Immanuel Kant.

Son los primeros pensadores cristianos quienes «adoptaron, o quizá también reinventaron, la nueva concepción de la dignitas de Cicerón como un atributo humano interno y en su núcleo inmutable, es decir, la gran dignidad humana» (p. 38). Avanza Von der Pfordten por el Renacimiento Italiano, destacando sobre todo la figura de Giovanni Pico Della Mirandola, a quién le es atribuible un programa de autoperfeccionamiento del hombre en su famoso Discurso pero aún no ligado al concepto de dignidad humana, pues en todo la Oratio Pico no usó ni una vez el concepto ni la palabra dignidad. Dejo a los lectores que descubran el riguroso análisis que despliega Von der Pfordten para sostener que Pico evitó el uso de la palabra y cómo fue su editor póstumo quien la coloca en el título de la obra. Ya en Pufendorf hay un tratamiento de la 'gran' dignidad desligada de su resonancia romana dignitas y, es más, un uso de la palabra dignatio que hace entender la dignidad como valía consagrando así la dignidad en su sentido 'intermedio' de posición social ligada con el sentimiento de autorrespeto, el sentido de la propia valía que el hombre tiene de sí (p. 49). Ya con Kant la dignidad alcanza su excelso alcance al concebirse como autonomía o autodeterminación, esto es, como la capacidad de darse cada uno su propia legislación moral (p. 53).

El concepto de dignidad humana fue apenas desarrollado por los filósofos alemanes que siguieron a Kant, como Fichte, Hegel y Schelling (p. 55) y, en no pocos casos, el empleo de la noción de dignidad volvió a cargarse de aquellas interpretaciones religiosas contra las que Kant había protestado por ser imposiciones heterónomas a la persona como fin -aunque él tampoco salió airoso por completo de aquella tarea secularizadora-. Von der Pfordten prosigue el capítulo con Schiller, Bentham, Schopenhauer, Nietzsche hasta Lasalle, en el que se puede encontrar, en su discurso de 1862 El programa para los trabajadores, la formulación de una 'existencia humanamente digna' entendida como el cuarto concepto parcial de la dignidad, esto es, como condición económica de la dignidad humana (p. 59). Se finaliza este capítulo mostrando los textos jurídicos en los que aparece por primera vez, a comienzos del siglo XX, consagrada la dignidad humana aunque aún sin fuerza vinculante: la Constitución de Weimar de 1919 y la Constitución de la República de Irlanda de 1937. Ya con la Carta de las Naciones Unidas de 1945 y la Declaración Universal de Derechos Humanos de 1948 la dignidad alcanza un importante significado político y jurídico. Desde entonces y hasta nuestros días, la dignidad humana ha sido reconocida en varias constituciones, así como en pactos y declaraciones a nivel regional y universal.

El tercer capítulo lo dedica Von der Pfordten a las concepciones de la dignidad humana. El punto de partida más convincente, y la concepción que él abraza, es la 
consideración de la dignidad como autodeterminación sobre los intereses propios. Ya sabemos que desde Kant ese atributo humano interno e inmutable que es la dignidad ha sido despojado de sus fundamentos metafísicos y religiosos. Esto es, se opone siempre autodeterminación a heterodeterminación. Para Von der Pfordten, conocido internacionalmente como representante del llamado 'individualismo normativo', únicamente los individuos son en sentido último seres éticamente relevantes. No lo son así los colectivos, como el clan, la raza, la nación, el estado o la sociedad, lo que supondría un colectivismo normativo (p. 77). Si solo los individuos son éticamente relevantes, entonces se plantea la cuestión de qué atributo de esos individuos es ético-normativamente relevante. Tras desechar una cantidad considerable de propuestas -el impulso de conservación de Hobbes, el placer o la utilidad de Bentham o Mill, los derechos de Nozick o Dworkin, el bienestar de Raz, las capacidades de Sen y Nussbaum o los acuerdos ficticios de Rawls o Habermas-, Von der Pfordten llega a la conclusión de que el atributo inmanentemente humano más relevante es «la real o, por lo menos, potencial autodeterminación sobre los propios intereses; esto es, la determinación de los propios intereses de primer o inferior orden a través de los deseos y fines de segundo o superior orden. Una parte esencial de nuestra autocomprensión consiste en nuestra autodeterminación sobre nuestros propios intereses» (p. 82).

Además de la gran dignidad humana de la autodeterminación sobre sus propios intereses, todos los seres humanos tienen también la pequeña e intermedia dignidad humana: el mantenimiento de la posición social, el autorrespeto y la protección contra la degradación o humillación (p. 88). La posición social del individuo se halla pues en la interacción de dos perspectivas: la autoevaluación -la autoestimay la heterovaloración -ya que la comprensión que uno tiene de sí mismo también está influida por el reconocimiento que recibimos de los demás-. Por lo tanto, son ataques a este 'subtipo' de dignidad las humillaciones de la exclusión y la marginación -aquí Von der Pfordten sigue en cierta medida el planteamiento de Avishai Margalit en La sociedad decente-. Lo que pone de manifiesto Von der Pfordten es si toda exclusión de grupos o asociaciones humanas supondrían una violación de la pequeña o intermedia dignidad humana. Pues, por ejemplo, «¿no está acaso autorizada una comunidad religiosa a enjuiciar por sí misma si alguien según su opinión es 'ortodoxo' y a excluirlo en caso de una respuesta negativa, sin que ello implique degradarlo, esto es, lesionar su pequeña dignidad?» (p. 91). Seguidamente, en la explicación de la concepción de la dignidad como condición económica, Von der Pfordten recurre a la jurisprudencia del Tribunal Federal alemán que ha reconocido una obligación estatal de protección de la existencia humanamente digna que engloba el deber de aseguramiento o mantenimiento de un mínimo existencial (p. 94).

El resto del capítulo tercero lo dedica Von der Pfordten para plantear interrogantes e ir contestándolos de tal modo que el cuadro final dibujado sobre las concepciones de la dignidad humana revelan una protección con propósito bastante amplio, abarcador y universalista. A las propuestas escépticas y a aquellas que ligan la dignidad a teorías del reconocimiento recíproco -Hofmann, Habermas-, Von der Pfordten las tacha de reduccionistas por negar precisamente aquello que la gran dignidad humana significa, a saber, la independencia de la autodeterminación de los seres humanos del reconocimiento social (p. 97). Se pregunta Von der Pfordten si la dignidad es una agrupación de intereses o derechos centrales (p. 99), afirmando en cambio la independencia de la dignidad de toda nómina de derechos -el catálogo de derechos no agota el concepto de la dignidad-; también se cuestiona si es una palabra vacía y prescindible, acaso una ilusión, lo que Von der Pfordten negará.

Continúa el filósofo alemán interrogándose sobre quién es el titular de la dignidad humana y su protección. Saldrán aquí cuestiones relativas al principio - 
aborto- y fin -eutanasia- de la vida humana. Para Von der Pfordten «los intereses de las generaciones futuras se deben pues tomar en cuenta en el presente, aunque los individuos humanos, como parte de esas generaciones futuras, todavía no existan o no sean todavía individualizables» (p. 104). Son los casos en los que la obligación de respetar la dignidad humana nos impone una acción con efecto diferido en el tiempo para su protección: pues la protección del atributo de la autodeterminación sobre los propios intereses del sujeto, nos exige proteger la dignidad incluso antes de la aparición real-fáctica del atributo. Sólo protegiendo hoy esa autodeterminación potencial, nos aseguraríamos que el sujeto la pueda detentar mañana. Debido a que esta protección se trata de una obligación y un derecho, no es posible ni necesario supeditarla a la configuración de un determinado estadio del desarrollo del embrión: «ya desde antes de la formación del embrión, su posterior autodeterminación sobre sus propios intereses está protegida y con su aparición tal protección se concreta respecto a él como individuo» (p. 106). Análoga estrategia se sigue para argumentar la extensión de la protección de la dignidad humana más allá de la muerte: la autodeterminación -interés de segundo orden- que se haya hecho en vida sobre intereses de primer orden -cómo debe ser tratado el cuerpo- obliga ética y jurídicamente para que los sobrevivientes cumplan la voluntad del fallecido y, con ello, respeten su dignidad. Lógicamente, tras la muerte de una persona, su gran dignidad, entendida como autodeterminación, fácticamente desaparece: no podrán aparecer más intereses de segundo orden y sólo son protegibles los intereses de primer orden que en base a aquella gran dignidad el causante determinó en vida. En esta misma línea continua Von der Pfordten argumentando a favor de la dignidad humana de aquellos que se hallan en coma profundo o sufren una grave alteración mental.

Von der Pfordten se mete en los procelosos jardines de la intangibilidad e inviolabilidad de la dignidad humana y sale, en mi opinión, bastante bien parado. Al ser humano no se le puede retirar su dignidad; aunque la dignidad sea susceptible de lesión, no es anulable por completo. La gran dignidad humana de la autodeterminación es fácticamente intangible pues, aun en situaciones extremas, como la tortura, la esclavitud, o el trabajo forzado, no puede ser despojada por entero. En estos casos de severa restricción, el ser humano se sigue manteniendo como un ser espiritual con facultad para la autodeterminación; aunque en ese momento concreto la facultad no pueda determinarse en intereses de primer orden, la capacidad para hacerlo sigue siendo inmanentemente suya. La dignidad puede ser violada, restringida, pero no arrebatada. Hay que terminar con un ser humano para terminar con todos sus atributos que comprenden su gran dignidad (p. 111). Sí, en cambio, resulta posible que la pérdida de la posición social conduzca prácticamente a la pérdida casi completa del autorrespeto; de la misma manera que una merma de los presupuestos materiales de la dignidad suponen un serio menoscabo de la condición económica de la misma. Por tanto, sólo la gran dignidad es la que resulta inviolable e intangible sin restricción.

El siguiente epígrafe de este capítulo versa sobre la prescriptiva prohibición de ponderación. Se pregunta Von der Pfordten si «¿exige la ética al derecho vigente la prescripción de la imponderabilidad de la dignidad humana?» (p. 123). Si bien los fundamentos éticos son prima facie también buenos fundamentos para el derecho positivo, sin embargo, no podemos concluir que todo lo que está éticamente justificado o moralmente exigido puede también ser razonablemente prescrito por el derecho de la misma manera. Algunos deberes morales, como la prohibición general de mentir, no pueden ser exigidos por el derecho. E, inversamente, hay derecho que no se corresponde con ninguna exigencia moral como, por ejemplo, la exigencia de notario para otorgar testamento válido. Llega Von der Pfordten a concluir que la gran dignidad es normativamente intangible para los órganos del Estado, esto es, no pueden ponderarla para proteger otros derechos fundamentales. Una restricción de la 
dignidad humana «no puede ser justificada bajo ninguna circunstancia por referencia a algún derecho fundamental individual, por ejemplo, la protección de la vida, la libertad religiosa, etc.» (p. 127).

Los siguientes apartados de este capítulo plantean una serie de interrogantes que son respondidos de tal forma que siempre se busca la máxima protección a la dignidad humana: ¿existe y obliga universalmente la dignidad humana?, ¿se puede renunciar a la dignidad humana?, ¿fundamenta la dignidad humana los derechos humanos?, ¿es la dignidad humana un derecho humano?, ¿reduce su propia dignidad quien vulnera la dignidad humana?

La obra se cierra con un capítulo dedicado a analizar casos actuales y controvertidos en los que entra en juego el papel de la dignidad humana en el derecho. Von der Pfordten muestra gran maestría para pasar del plano teórico al práctico y así va desplegando en cada argumentación, en base a la concepción teórica de la gran dignidad como autodeterminación, una respuesta pormenorizada a problemas reales de nuestros días que retan y desafían la autonomía de los individuos: así, por ejemplo, la cadena perpetua viola la gran dignidad humana, pues pese al mantenimiento de la capacidad de autodeterminación -segundo orden-, esa capacidad no se puede materializar en el logro de deseos y fines ya que el preso no tendrá nunca oportunidad real de ser liberado para llevarlos a cabo; también la alimentación forzosa del individuo consciente supondría una subordinación de su determinación individual de protestar bajo esa manifestación -cuestión distinta es el papel de garante que asume el Estado cuando el individuo pasa a estar en un estado de inconsciencia derivado de aquella decisión-. Von der Pfordten muestra la infracción de la dignidad que se comete cuando el Estado emplea detectores de mentiras o cuando derriba aviones secuestrados por terroristas poniendo con ello en riesgo la vida de otros individuos a bordo de la aeronave, que pasarían a ser medio para otros fines. Las últimas cuestiones que responde Von der Pfordten proceden del ámbito de la bioética (pp. 143 y ss.): ¿Vulneran el diagnóstico genético preimplantacional y la selección de la dignidad humana?, ¿se pueden modificar genéticamente los seres humanos? y, por fin, ¿se pueden clonar seres humanos? Dejemos al lector que halle las respuestas en este estimulante y fructífero libro de Von der Pfordten, en el que la defensa de la dignidad humana, con todo su potencial y fuerza moral, es la única y auténtica protagonista. 